\title{
Modeling and simulation of the effects of landslide on circulation of transports on the mountain roads
}

\author{
Manh Hung Nguyen ${ }^{1,2}$ \\ ${ }^{1}$ Posts and Telecommunications Institute of Technology (PTIT) \\ ${ }^{2}$ UMI UMMISCO 209 (IRD/UPMC), Hanoi, Vietnam
}

\author{
Tuong Vinh $\mathrm{Ho}^{2,3}$ \\ ${ }^{3}$ IFI, Vietnam National University in Hanoi \\ ${ }^{2}$ UMI UMMISCO 209 (IRD/UPMC), Hanoi, Vietnam
}

\begin{abstract}
Landslides, as one of the major natural hazards, account each year for enormous property damage in terms of both direct and indirect costs. Mountain roads where probability of land sliding is the highest, causes hurdles not only in the traffic flow but generate various traffic problems in the form of congestion, high accidents rate and waste of time. This paper introduces an agent-based model for modeling and simulation of the effects of landslide on the circulation of transports on mountain roads. This model is applied to the National Road $\mathrm{N}^{\circ} 6$ of Vietnam to visualize and analyze the effects of landslide on the road when it occurs. This model could help us to improve and optimally organize of landslide warning and rescue system on the road.
\end{abstract}

Keywords-Simulation model, Traffic network simulation, Landslide effect, Multiagent system

\section{INTRODUCTION}

Landslides, as one of the major natural hazards, account each year for enormous property damage in terms of both direct and indirect costs. Mountain roads where probability of land sliding is the highest, causes hurdles not only in the traffic flow but generate various traffic problems in the form of congestion, high accidents rate and waste of time. As the others extreme conditions, landslides are rare and high-impact events and, thus, are difficult to manage. The difficulties arise from the following:

- They are rare and, as a result, there is a lack of data to help analyzing the mechanisms of the emergencies and the responses of the real world systems.

- They are non-recurrent, so management strategies developed in response to these extreme conditions may hardly be used in practice, and thus it is difficult to evaluate their effectiveness.

- They are disruptive and destructive, so it might be prohibitive to physically replicate these conditions for learning and training purposes.

As a low-cost, safe, non-disruptive, reproducible, and testable means of problem solving, modeling and simulation is particularly attractive in addressing problems under extreme conditions like landslides. In this report, we only concentrate on the landslide impacts after they happen, but not how do they happen. Modeling and simulation of how the landslide occurs based on the underground structure, geology, rainfall and storms, etc. in the mountain region is thus out of the scope of this report.

Concretely, we are interesting in landslides impacts on circulation on mountain roads. We consider the modelling and simulation of the effects of landslide on circulation of transports on mountain roads as a kind of transportation network simulation problem. In which, the landslide is considered as a cause of obstacles on road that the transports have to avoid during their circulation. And the possibility of occurrence of landslide at a point on a road is considered as an input data for the model we propose. We concentrate on the effects of landslide after it happens, but not how does it happen. The modelling and simulation of how the landslide occurs based on the underground structure, geology, rainfall and storms, etc. in the mountain region is thus out of the scale of this paper.

Recently, there have been many researches interested in the field of transportation network simulation. Therefore, there have been many models and tools proposed. Most of them are agent-based models. In which, intelligent agent and multiagent system seem to be suitable for simulate transportation network at the micro level. Each transport is thus modelled as an intelligent agent. It could observe other transports and obstacles to change its own speed as well as direction to go to its destination as fast as possible. The transportation network therefore could be modelled as a multiagent system whose each agent has a personnel goal (its destination to go) and they have to coordinate and/or interact together in order to prevent accidents from happening. There are many models proposed in this tendency. For instance, AgentPolis [12] is a fully agent-based platform for modeling multi-modal transportation systems. It comprises a high-performance discreteevent simulation core, a cohesive set of high-level abstractions for building extensible agent-based models and a library of predefined components frequently used in transportation and mobility models. MATSim development team [6] is developing a framework and platform for a transportation network simulation, called MATSim. MATSim provides a toolbox to 
implement large-scale agent-based transport simulations. The toolbox consists of several modules which can be combined or used stand-alone: toolbox for demand-modeling, agent-based mobility-simulation (traffic flow simulation), re-planning, a controller to iteratively run simulations as well as methods to analyze the output generated by the modules. SUMO (Simulation of Urban MObility) [4], [13] is a highly portable, microscopic road traffic simulation package designed to handle large road networks. It is mainly developed by employees of the Institute of Transportation Systems at the German Aerospace Center. SUMO allows to simulate how a given traffic demand which consists of single vehicles moves through a given road network. The simulation allows to address a large set of traffic management topics. It is purely microscopic: each vehicle is modelled explicitly, has an own route, and moves individually through the network. The V2X simulation runtime infrastructure - VSimRTI [25] - enables the preparation and execution of V2X simulations. It is a flexible system which simulates traffic flow dynamically. VSimRTI couples different simulators, thus, allowing the simulation of the various aspects of future intelligent transportation systems. Al-Dmour [1] developed TarffSim, a Multiagent Traffic Simulation for micro-simulation and macro-simulation of traffic. TraffSim was implemented by using NetLogo. Balakrishna et al. [2] presented a simulation-based framework for the modeling of transportation network performance under emergency conditions. The system extends the well-established dynamic traffic assignment (DTA) framework. Barcel and Casas [3] discussed some of the most critical aspects of the dynamic simulation of road networks, namely the heuristic dynamic assignment, the implied route choice models, and the validation methodology, a key issue to determine the degree of validity and significance of the simulation results. Caris et al. [5] proposed a discrete event simulation methodology to understand the network dynamics and analyze policy measures with the intention of stimulating inter-modal barge transport. The simulation model allows to quantify a number of network properties resulting from the interaction of freight Frick [9] dealt with the flexible implementation of transportation networks into a simulation model and realizing scenarios. Gokulan and Srinivasan [10] have been implemented two different types of multi-agent architectures on a simulated complex urban traffic network in Singapore for adaptive intelligent signal control. Holmgren et al. [11] presented the Transportation And Production Agent-based Simulator (TAPAS), which is an agent-based model for simulation of transport chains.Lotzmann [14] presented an agent-based traffic simulation approach which sees agents as individual traffic participants moving in an artificial environment. Meignan et al. [16] presented a bus-network simulation tools which include these specificities and allows to analyze and evaluate a bus-network at diverse space and time scales. They adopted a multiagent approach to describe the global system operation as behaviors of numerous autonomous entities such as buses and travelers. Mengistu et al. [17] provided a framework for development and execution of parallel applications such as multi-agent based simulation (MABS) in large scale. In the work of Manning et al. [15], a mathematical model is given for the system failure and a statistical model is formulated for the joint distribution of rainfall at different points along the railway line. Perumalla [22] designed a simulator with memory and speed efficiency as the goals from the outset, and, specifically, scalability via parallel execution. The design makes use of discrete event modeling techniques as well as parallel simulation methods. Piorkowski et al. [23] are developing TraNS, an open-source simulation environment, as a necessary tool for proper evaluation of newly developed protocols for Vehicular Ad Hoc Networks (VANETs). Ramos et al. [24] described the main features of contemporary ITS, emphasizing the Portuguese case, and describes the fundamental modeling \& simulation tools that are considered critical to support the daily operation of the urban transportation system. Xu and Tan [26] introduced a hyper graph-based offline road network partitioning solution, which is suitable for future distributed transportation simulations with ITS applications. Zacharewicz et al. [27] dealt with the development of this simulation platform, based on Generalized Discrete Event Specification (G-DEVS) models and HLA (High Level Architecture) standard. Zhang et al. [28] presented an agent-based discrete-event simulation (AB-DES) modeling approach for transportation evacuation simulation based on a hybrid continuous and cell space. Zhang and Lv [29] presented a multi-agent competition model to describe the competition relationship in integrated transportation system.

In Vietnam, there are also some researches on modelling and simulation of urban transportation network. For instances, Nguyen and Ho [18] proposed an agent-based model for modelling and simulation the Vietnamese behavior in circulation in the city of Hanoi. Nguyen et al. [19], [20], [21] also proposed an agent-based model for simulation of urban transportation network.

Our objective thus is to develop a simulation model and tool to visualize the effects of landslide on circulation of transports on the mountain road. The model could be applied not only for the National Road $\mathrm{N}^{\circ} 6$ of Vietnam but also be applied for any mountain road in Vietnam. This tool also aims to help the mountain road management office to improve and optimise the organisation of landslide rescue center along the roads to reduce the waiting time of transport as well as the resources needed (rescue machine, communication, energy) to clean and repair the roads once landslide occurs.

This paper is organised as follows: Section II presents our agent-based model of the system. Section III presents a case study in which we apply the proposed model to simulate the effects of landslide on the National Road $\mathrm{N}^{\circ} 6$. Finally, section IV discusses the presented work and draws some perspectives for future work.

\section{MOdelling OF THE SYSTEM}

This section presents the modelling of the system. The system is based on multi-agent system. We thus model find kinds of agents (Fig. 1):

- Road agent: The agent represents the road

- Landslide point agent: The agent represents the points where the landslide occurs.

- Transport agent: The agent represents the transport on the road

- Rescue center agent: The agent represents the emergency landslide rescue center. 


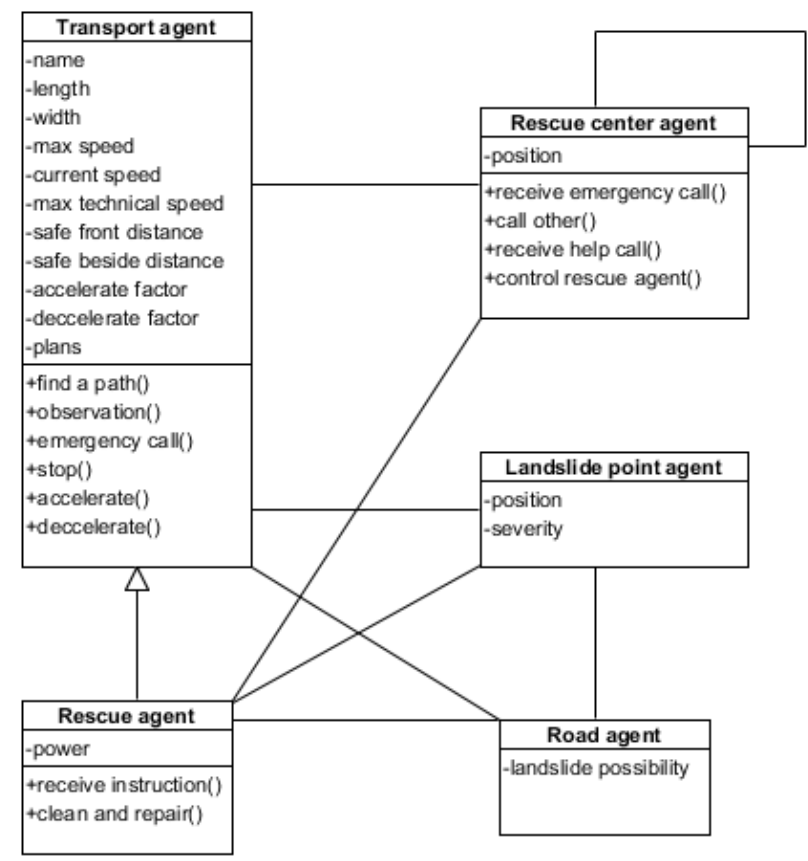

Fig. 1: Modelling of agents in the system

- Rescue agent: The agent represents the emergency rescue machine to clean and repair the road where the landslide occurs.

These agents will be described in detail in the following sections.

Note that in this model, we do not model an object in the transportation network, that is traffic light. The reason is that the traffic light has important effects in the circulation of transport in urban city circulation simulation scale: the average circulation time of transport, the average waiting time at intersections of transport could be affected by the change of traffic light operation policy when we consider and simulate the system in a small scale as at an intersection (see Nguyen et al. [20]), or in a city (see Nguyen et al. [18], [19]). However, when we consider the system in a large scale as a whole along a road on the mountain, the length of road could reach hundreds kilometres, the waiting time at intersections caused by traffic light is so small, in regarding the whole time to pass the road, that we do not need to model the traffic light in this model.

\section{A. Road agent}

Road agent represents the real road. The real data is got from GIS file (Geographical Information System). So this kind of agent has all geometry attributes: position, length, etc. Each agent represents a short segment of the road.

This kind of agent has no behavior.

The most important attribute of this agent is its position because this attribute is related to the potential landslide at that position. So we need to model an additional attribute for this agent, that is:
- landslide possibility: the possibility to occur the landslide at that segment of road.

\section{B. Landslide point agent}

landslide point agent represents the points at that the landslide occurs. This agent has following attributes:

- position: the position where the landslide occurs.

- severity: the level of severity of landslide, or the amount of stone slides on the road. The higher the severity is, the longer time the rescue agent need to rescue the road to get normal circulation status.

This agent has no behavior. It will be died when the rescue agents finish their work at that point.

\section{Transport agent}

A transport could be a trunk, a bus, a car (including taxi), a motor. It has some attributes, behaviors and ability to move. So we need to model it as an agent in the system. Because a driver is assigned to his transport so we consider the whole of a driver and his transport as an unique transport agent.

1) Attributes: A transport has these attributes:

- name: name of a transport.

- length (denoted as l): real length of a transport.

- $\quad$ width (denoted as $d$ ): real width of the transport.

- max speed (denoted as $v_{\max }$ ): the maximal allowed speed, for the transport, by law

- current speed (denoted as v): the current speed of the transport

- max technical speed (denoted as $v_{\text {tech }}$ ): the maximal technical speed of a transport, limited by the engine of the transport.

- $\quad$ safe front distance (denoted as $d_{f}$ ): the minimum distance to the nearest transport in front that keep safe for circulation. This distance is estimated by following formula:

$$
d_{f}=\frac{l * v}{v_{\max }}+\theta
$$

where $\theta$ is the minimum distance allowed among transports when stopping.

- $\quad$ safe beside distance (denoted as $d_{b}$ ): the minimum distance to the nearest beside transport that keep safe for circulation. This distance is estimated by following formula:

$$
d_{b}=\frac{d * v}{v_{\max }}+\theta
$$

- accelerate factor (denoted as $\alpha$ ): the ability to speed up in an unit of time

- decelerate factor (denoted as $\beta$ ): the ability to slow down in an unit of time

- A set of circulation plans. A plan contains following information: 
- start time: the start time to circulate

- department: the start position of the circulation. A position is represented by its real (longitude $(\mathrm{x})$, latitude $(\mathrm{y})$ ).

- destination: the destination(s) to get to of the individual

- max speed: the maximal speed for the individual. This must not be higher than the maximal permitted speed by law.

- type of vehicle: this attribute is reserved to determine the size of vehicle on the road.

\section{2) Behavior: Behavior of transport agents:}

- find a path: this will find a path to go. A transport agent finds a path when: either it starts a new plan; or it want to change the path when it is blocked somewhere on the way. A path is simply determined by the Dijkstra's algorithm (Dijkstra [7]) on a graph constructed from the road as follow:

- Each intersection forms a node of the graph.

- Each road forms an arc with the same direction. If a long road has many segment points to change the direction, then each segment of road (between two consecutive segment points) will form an arc with the same direction.

- The weight of each arc is proportional to the length of the corresponding segment of road.

- observation: this is an action of the driver of a transport, including of observation of the landslide, observation of obstacles.

- $\quad$ emergency calling: the transport agent faces a landslide, it will call to one of rescue center agents to alert the occurrence of landslide.

- $\quad$ stop: a transport agent stops at a blocked point caused by landslide.

- accelerate: a transport agent will accelerate when: (1) there is no obstacles in safe front distance and safe beside distance of it; and (2) its speed does not reach the max speed yet. The new speed will accelerate to:

$$
v_{t+1}=\min \left\{v_{t}+\alpha, v_{\max }\right\}
$$

where $v_{t}, v_{t+1}$ are the speed of the transport agent at the simulation step $t, t+1$, respectively.

- decelerate: a transport agent has to decelerate when: (1) there is some obstacles in safe front distance or safe beside distance of it; or (2) it faces a rescue agent; or (3) it intends to stop. The new speed will decelerate to:

$$
v_{t+1}=\max \left\{v_{t}-\beta, 0\right\}
$$

The transition among behaviors of a transport agent is depicted in the Figure 2: A transport agent starts his movement by starting a plan on time. Firstly, it finds a path to go. During moving, it observes three kinds of objects: the landslide, the destination, and the obstacle. In observing the landslide: if there is some landslide occurs, it will stop, take an emergency call to one of rescue center agent, and may be re-find the path; otherwise, it will continue to move. In observing the obstacle,

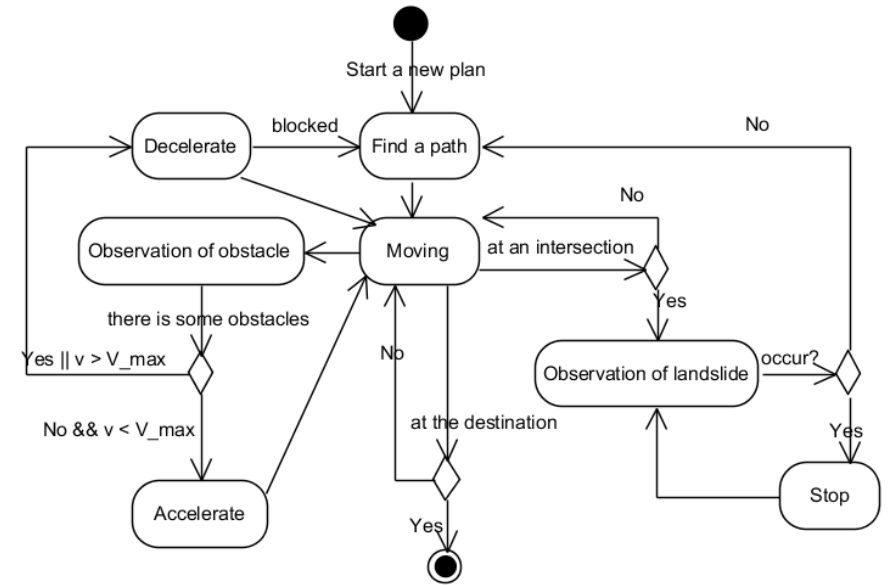

Fig. 2: Behaviors of transport agent

it will accelerate if there is no obstacle and its current speed $v$ is still lower than the allowed speed $v_{\max }$. It will decelerate if there are some obstacles or its speed $v$ is already higher than the allowed speed $v_{\max }$ (for the case of obey transport). Otherwise, it continues to move with the current speed. Note that, the circulation priority of transport agent is lower than that of rescue agent, so it have to decelerate and release the road for rescue agent. In observing the destination, if it is at the destination, it finishes the circulation. Otherwise, it continues to move.

\section{Rescue center agent}

Rescue center agent represents the emergency landslide rescue center. This agent plays the role of managing a set of rescue agents. They are also able to communicate with each others to optimally control rescue agents if it is necessary.

\section{1) Attribute:}

- position: the position where it is situated.

2) Behavior:

- receiving of emergency calls: this agent is responsible for receiving the emergency call from transport agents to be aware the occurrence and the position of landslide.

- call other: In the case of there is not enough rescue agents, or it is not optimal if using its own rescue agents, then the rescue center agent could call other rescue center agents to ask for help.

- $\quad$ receiving of helping calls: this agent is also able to receive the helping call from other rescue center agents.

- control rescue agent: after receiving emergency call from transport agent or helping call from other rescue center agents, it could control some of its rescue agents to go to the point where the landslide occurs.

The transition among behaviors of a rescue center agent is depicted in the Figure 3: It is always in the status of waiting. When it receives a call (emergency call from transport agent, 


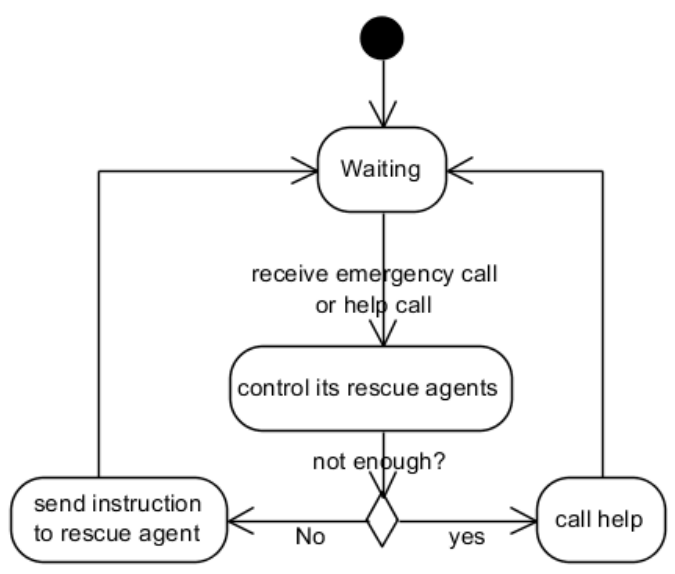

Fig. 3: Behaviors of rescue center agent

or helping call from other rescue center agent), it will start the control process by considering the number of rescue agent that it owns. If the number is not enough for rescue propose, it will helping call to other for requirement of some help. Otherwise, it sends instruction to selected rescue agents to put them into mission. After all, it returns to the permanent status of waiting for the next call.

\section{E. Rescue agent}

Rescue agent represents the emergency rescue machine when the center of landslide rescue receives the landslide occurrence news. This agent is basically the same with transport agents on the attributes and the behaviors to move. Moreover, this has some more attributes and behaviors.

\section{1) Additional attribute:}

- power: the ability to repair and clean the road after landslide. The more powerful it is, the faster the road is rescued.

\section{2) Additional behavior:}

- instruction receive: rescue agent is under the control of the rescue center agent. So it is able to receive and treat instructions from rescue center agent. The instruction may be one of these kinds: start to move toward the landslide position, start to repair and clean, pause (repair and clean), return to a rescue center.

- repair and clean: when arriving the point of landslide, it cleans the rolled stones and repairs the road. When it finishes its work, the road is through and the rescue agent returns to the center of rescue.

The transition among behaviors of a rescue agent is depicted in the Figure 4: The rescue agent is always in the status of waiting at one of rescue center agents. When it receives an instruction from the rescue center agent, it will start to move to the reported landslide point agent. During this movement, it acts as any kind of transport agent with the highest priority of circulation on the road. When it arrives at the landslide point agent, it starts to clean and repair the effected road agent. The time to clean and repair depends on the relation between the

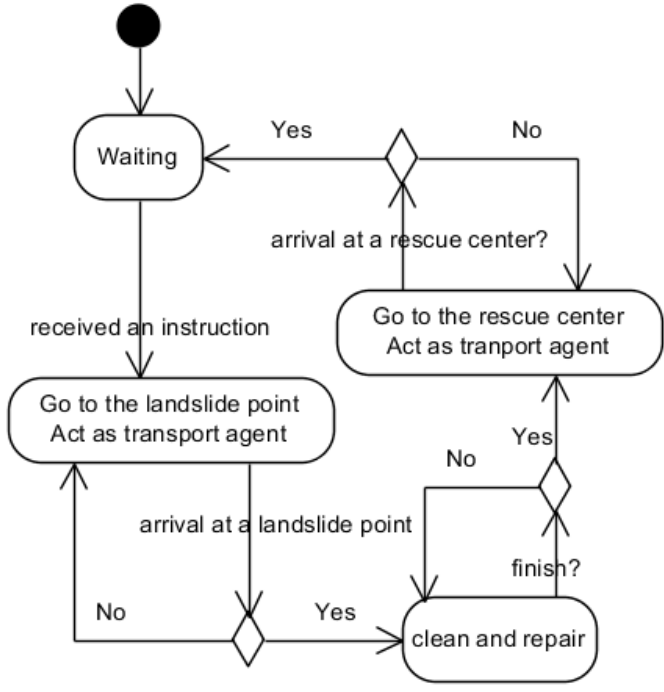

Fig. 4: Behaviors of rescue agent

severity of landslide point agent and the total of power of all rescue agents in the mission. After finishing the cleaning and repairing the effected road agent, the rescue agent returns to its original rescue center agent. During this movement, it also acts as any kind of transport agent with normal priority of circulation on the road. When it arrives at the rescue center agent, it returns to the permanent status of waiting for the next instruction.

\section{F. A standard scenario}

This is a scenario of the simulation:

1 All transport agents are normally moving on the road to their target.

2 There is some landslide point agent occurs at some points on the road

3 The road agent is blocked at that point.

4 The first transport agent faces the landslide point agent calls one of rescue center agents. It is also blocked at that point.

5 The called rescue center agent calls some rescue agent to go to the blocked point. In some variant case, the rescue center agent could call other rescue center agents to have more rescue agents if it is not enough or for the reason of optimisation of waiting time.

6 The selected rescue agent goes to the blocked point after receiving the instruction from rescue center agent.

7 When the rescue agent arrives at the blocked point, it stats to clean the stones from the road and then repair the road. The time taken for this operation depends on the severity of landslide agent, the power and the number of rescue agent.

8 During the time of cleaning and repairing of rescue agent, all transports agents passing the blocked point 
are still blocked, therefore the waiting time of each one continuously increases.

9 When the blocked point is cleaned, the landslide agent dies, and the blocked transport agents continue to go to their target (their waiting time stops counting)

10 The rescue agent returns to its original rescue center agent.

We would like to display this scenario on the visualization level of the simulation.

\section{A CASE STUDY: SIMULATION OF THE LANDSLIDE'S EFFECT ON CIRCULATION OF THE NATIONAL ROAD $\mathrm{N}^{\circ} 6$}

This section applies the proposed model to simulate the effects of landslide on the circulation on the National Road $\mathrm{N}^{\circ} 6$ of Vietnam (in the North-West of Vietnam). Section III-A presents the modelling and simulation of the system. Section III-B presents the obtained simulation results.

\section{A. Simulation setup}

1) Input data: The input data for the simulation is set as follow:

- Roads map: The National Road $\mathrm{N}^{\circ} 6$ is situated in the North-West of Vietnam. We extracted its data as a GIS file (Fig.5.a,b). It is considered from Hoa Binh city (attitude: $20.789 \mathrm{~N}$, longitude: 105.342E) to Son La city (attitude: $21.325 \mathrm{~N}$, longitude: 103.915E).

- Landslide possibility along the road: This data is provided by The Department of Geology, The Hanoi National University. In which, there are 41 potential point of landslide occurring, with different level, along the National Road $\mathrm{N}^{\circ} 6$ (Fig.5.c). We normalise the occurring potential of each point by a number in the interval $[0,1]$ : the more the value is high, the more the possibility of landslide occurs at that point is high.

- Transport distribution and plans: We refer this data from the statistic data of the National Center of Statistic taken in 2009. On basing on this data, we estimate the number of permanent transport is about $500-1000$ on the whole road at any daily moment, the average speed is about $25-50 \mathrm{~km} / \mathrm{h}$.

- $\quad$ Landslide rescue centers: We estimate that each big town of each city along the whole National Road $N^{\circ} 6$ has at least a rescue center, and each center has at least a rescue machine (Fig.5.d).

2) Analysis and evaluation criteria: At the output of simulation, we need to calculate the following parameters:

- Number of transports blocked: The number, in total, of transports which are blocked at the point where the landslide occurs.

- Total waiting time of each blocked transport: The total waiting time that each transport wasted in being blocked at the point where the landslide occurs.

These parameters help us to compare the effect of different rescue solutions when the landslide occurs. By reducing the

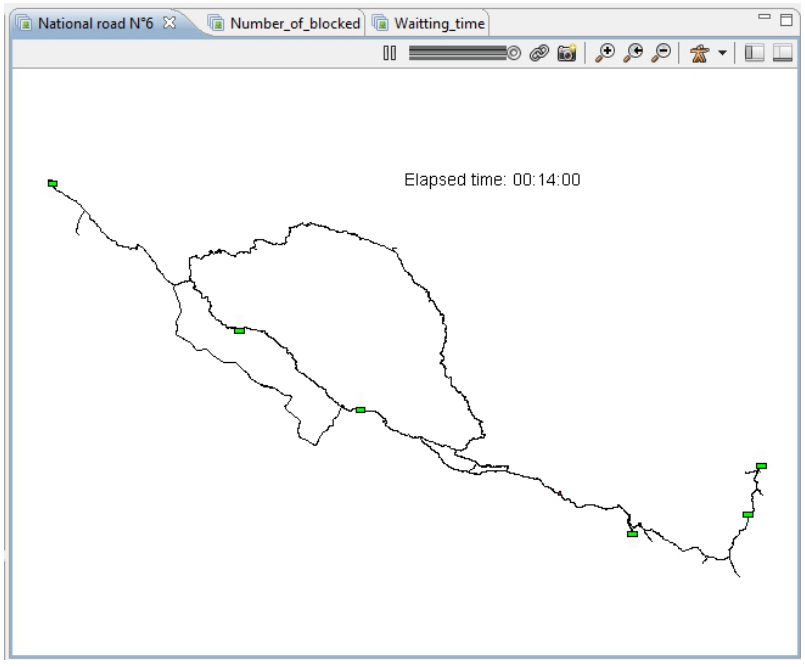

(a) Full view

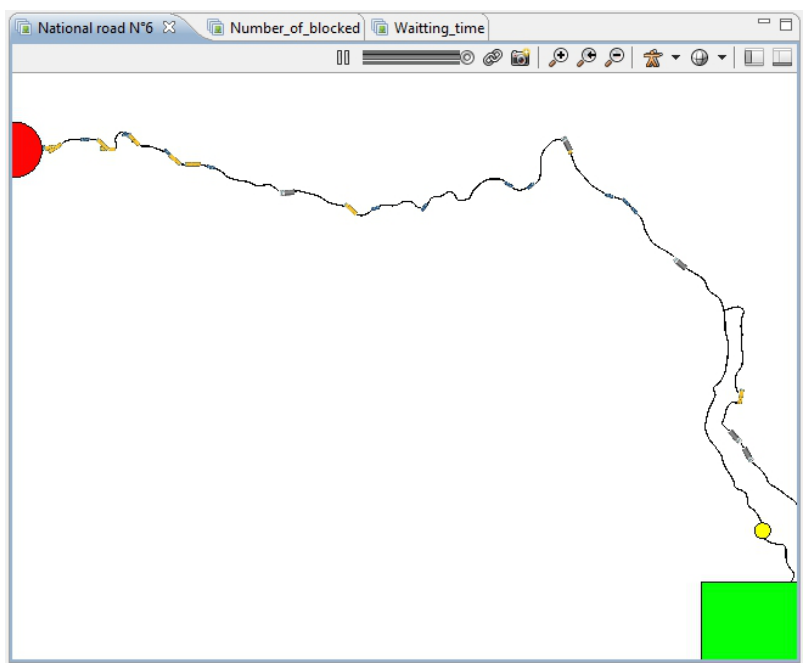

(b) A zoomed view

Fig. 6: Visual results of the simulation

value of these parameters, we could improve and optimise the organisation of landslide rescue system.

3) Simulation platform: Our simulation of the effects of landslide on circulation on the National Road $\mathrm{N}^{\circ} 6$ of Vietnam is implemented in the simulation platform GAMA [8]. GAMA is integrated and generic tools to support the representation of features usually associated with real complex systems, namely rich, dynamic and realistic environments or multiple levels of agency. It allows modelers, thanks to the use of a highlevel modeling language, to build, couple and reuse complex models combining various agent architectures, environment representations and levels of abstraction.

\section{B. Results}

In this section, we presents the simulation results at two levels: visualisation level, and statistic analysis level.

1) Visualisation of the system: At the visualisation level, the global view of the system is depicted in Fig.6.a. Because 


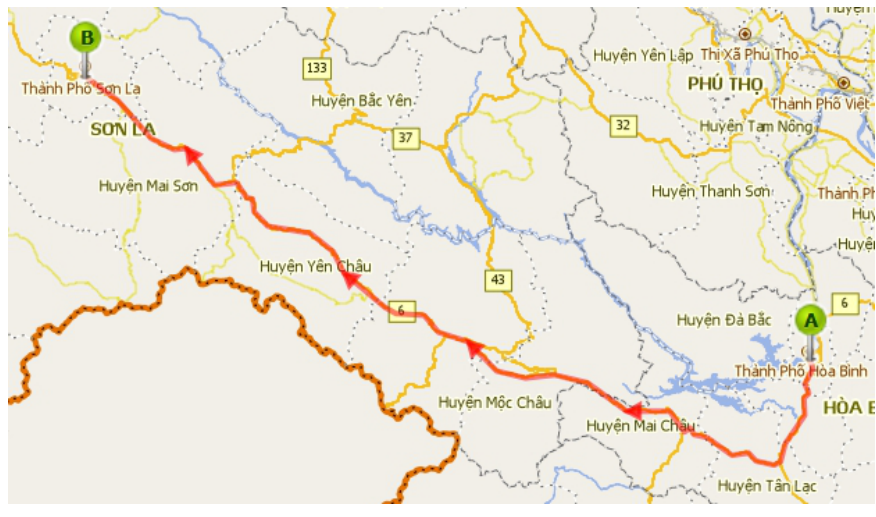

(a) Natural map

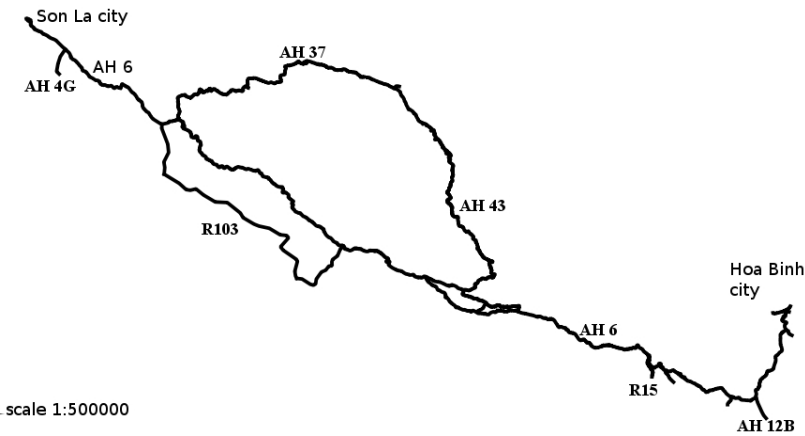

(b) Main road map

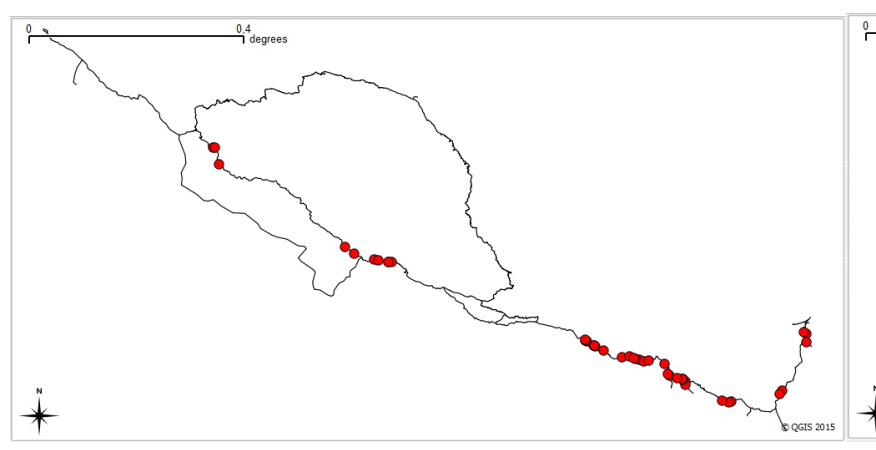

(c) Landslide points map

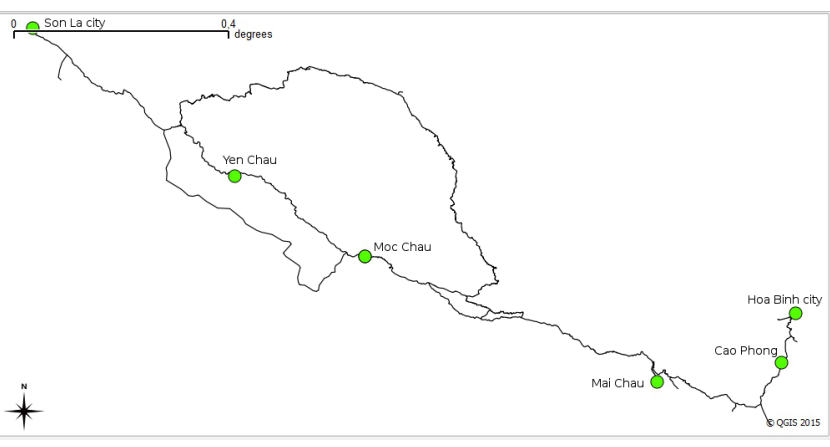

(d) Landslide rescue centers map

Fig. 5: Input GIS data for simulation

the big scale of the system, it is not easy to see all kind of agent in the system except we have to zoom it in detail. Fig.6.b shows in detail all kinds of agent in the system.

2) Statistical analysis: At the statistical analysis level, the simulation also displays the variance of output parameters by time: number of blocked transport, and total waiting time of blocked transport.

Fig.7.a depicts the number of blocked transport during simulation time. As we start to simulate the system at the moment when the landslide occurs, the number of blocked transport is regularly increased along the simulation time until the moment when the Rescue agent finishes its work and the road is cleaned and repaired. At that moment, the number of blocked transport is immediately down to zero.

In the same principle for the total waiting time of blocked transport, as depicted in Fig.7.b: The value of this output parameter is also increased along the simulation time until the moment when the Rescue agent finishes its work and the road is cleaned and repaired. From that moment, the total waiting time of blocked transport is no change because there is no more any blocked transport in the system.

By analysing the output parameters, we could compare the effect of different rescue solutions when the landslide occurs. The more the solution brings a lower value of these parameters, the more the solution is better.

3) Experiment 1: The effects of rescue agent power: The object of this experiment is to evaluate the importance of power
TABLE I: The effects of rescue agent power on output parameters

\begin{tabular}{|l|c|c|c|c|c|}
\hline Scenario & S1 & S2 & S3 & S4 & S5 \\
\hline Avg. Power & 1.5 & 2.0 & 2.5 & 3.0 & 3.5 \\
\hline Number of rescue agent & $7 / 7$ & $7 / 7$ & $7 / 7$ & $7 / 7$ & $7 / 7$ \\
\hline Blocked vehicle & 42 & 32 & 26 & 21 & 15 \\
\hline Total blocked time (m) & 2687 & 1830 & 1418 & 995 & 886 \\
\hline Avg. blocked time (m/vehicle) & 64 & 57 & 55 & 47 & 59 \\
\hline
\end{tabular}

of rescue agent on the number of blocked point and waiting time. We suppose that the higher the power of rescue agent, the shorter waiting time and the fewer number of blocked points we have. By default, the minimum portion of the power of a rescue agent in regard with the severity of landslide is $0.125 \%$ (The power is 1.5 , the severity is 1200 ). In order to evaluate this hypothesis, five scenarios have been performed, in which the severity is fixed while the power is changed from 1.5 to 3.5 .

The results of this experiment are depicted as in Tab.I. The number of blocked transport and also the waiting time decrease regularly when the power of rescue agent increases.

4) Experiment 2: The effects of vehicle kinds: In a transportation problem, the distribution of different kind of vehicle cans influent to the status of overall system. In this experiment, the ratio of distribution of car/bus/truck has been changed to evaluate how it affects to the number of blocked transports and waiting time. Three scenarios have been performed, in which the ration is changed. 


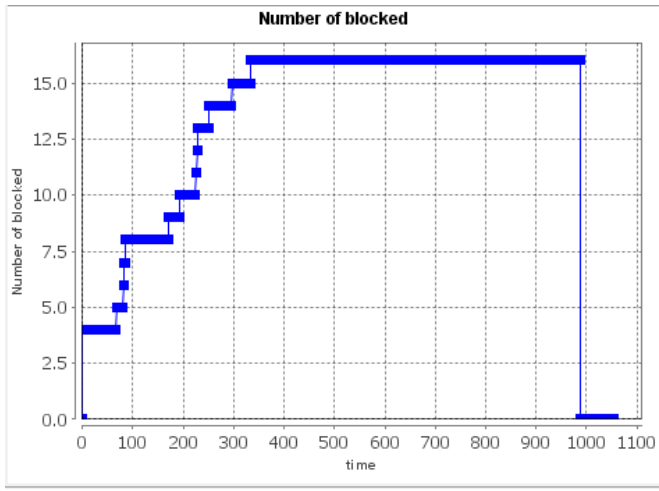

(a) Number of blocked vehicle

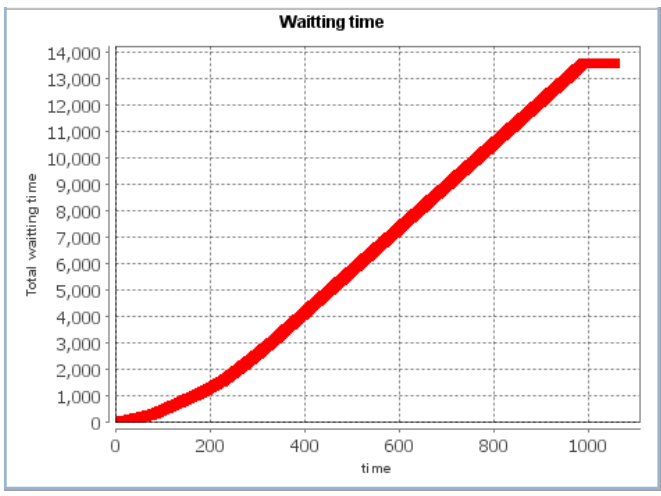

(b) Total blocked time

Fig. 7: Statistic results of the simulation

TABLE II: The effects of vehicle kind ratio on output parameters

\begin{tabular}{|l|c|c|c|}
\hline Scenario & S1 & S2 & S3 \\
\hline Car/bus/truck ratio (\%) & $50 / 30 / 20$ & $30 / 20 / 50$ & $20 / 50 / 30$ \\
\hline Blocked vehicle & 28 & 30 & 38 \\
\hline Total blocked time (m) & 1557 & 3000 & 2865 \\
\hline Avg. blocked time (m/vehicle) & 56 & 100 & 75 \\
\hline
\end{tabular}

The Tab.II illustrates the results of this experiment about the affect of different kind of vehicles. Three scenarios have been hold. For the first one, the number of blocked transport is less than the two other cases, while the waiting time is less in compare with the rest. This is explained by the size of each vehicle; in our experiment, the size of bus and truck is equal, and greater $60 \%$ with the size of car. Thus, the more number of bus and truck is, the higher waiting time is.

5) Experiment 3: The effects of the number of landslide points: In these experiments, we change only the number of landslides, and their position, the other parameter is fixed. The

TABLE III: The effects of the number of landslide points on output parameters

\begin{tabular}{|l|c|c|c|c|c|c|}
\hline Scenario & S1 & S2 & S3 & S4 & S5 & S6 \\
\hline Number of landslide & 1 & 2 & 3 & 4 & 5 & 6 \\
\hline Blocked vehicle & 8 & 18 & 30 & 60 & 62 & 73 \\
\hline Total blocked time (m) & 697 & 1250 & 2732 & 5033 & 9102 & 12718 \\
\hline Avg. blocked time (m/vehicle) & 87 & 69 & 91 & 84 & 147 & 174 \\
\hline
\end{tabular}

TABLE IV: The effects of the number of landslide position on output parameters

\begin{tabular}{|l|c|c|c|c|c|c|c|}
\hline Scenario & S1 & S2 & S3 & S4 & S5 & S6 & S7 \\
\hline Landslide in group & 1 & 2 & 3 & 4 & 5 & 6 & 7 \\
\hline Blocked vehicle & 14 & 28 & 30 & 69 & 19 & 33 & 20 \\
\hline Total blocked time (m) & 1437 & 1901 & 3300 & 12499 & 719 & 2692 & 1522 \\
\hline Avg. blocked time (m/vehicle) & 103 & 68 & 110 & 181 & 38 & 82 & 76 \\
\hline
\end{tabular}

number of landslide varies from 1 to 6 .

The results of experiment are shown in the Tab.III. As we start to simulate the system at the moment when the landslide occurs, the number of blocked transport has is increased when the number of landslide increases. The increasing amount between two consecutive experiments is quite significant.

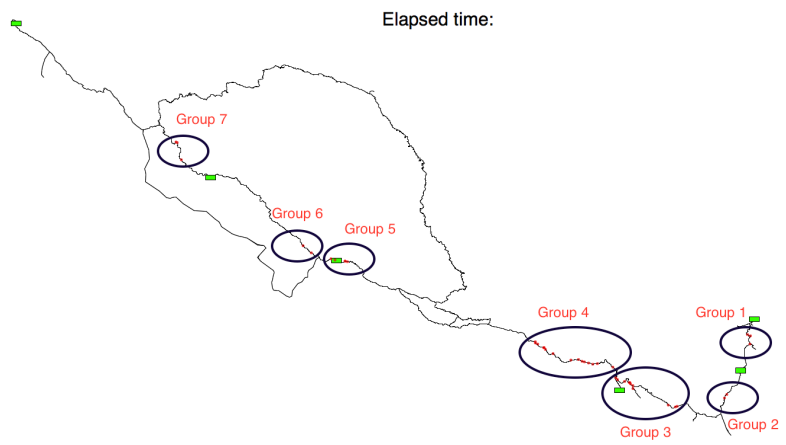

Fig. 8: Regrouping landslide points into seven groups

6) Experiment 4: The effects of landslide position: Actually, we have 41 potential landslides along the National Road $\mathrm{N}^{\circ} 6$. With different landslide positions, are we have different affect on the waiting time and number of blocked transport? In this experiment we will examine it. In order to separate the position of landslide, we regroup 41 landslide points into seven groups as depicted in Fig.8. And then, we examine seven scenarios: each scenario has a landslide in each group.

The results of this experiment are depicted in Tab.IV. Based on it, we can divide 4 groups. The first one contains the position of group 1, 5 which have the lowest waiting time and number of blocked transports; Next, it is the group 6, 7 in which the number of blocked transports and waiting time is greater that the first one; The third one is at the position of group 2,3; The last one is the position at group 4 where the number of blocked points and the waiting time are the worst.

At the position of group 4, there is only a route; no detour to avoid this one, so that when landslide occurs here, the number of blocked transports and the waiting time are the highest. The landslides at the group 1 are situated between two rescue centers; while the group 5 is very closed to a rescue center. Moreover, the position at the group 1 is near by the end point of the National Road $\mathrm{N}^{\circ} 6$, thus the quantity of vehicles is small. So that, when the landslide occur, the rescue agent can resolve it fast.

\section{CONCLUSION}

This paper proposed an agent-based model to model and simulate the effects of landslide on the circulation of transports 
on the mountain roads. In which we modeled five kinds of agent: Road agent, Landslide point agent, Transport agent, Rescue center agent, Rescue agent, and then simulated their behavior. The model is applied to simulate the effects of landslide on the circulation of transports on the National Road $\mathrm{N}^{\circ} 6$ of Vietnam. This is an useful tool for visualization the effects of landslide on the road.

Simulating to optimize some routing strategies when the landslide occurs or optimise the organisation of landslide rescue center network on the road are our works in the near future.

\section{ACKNOWLEDGMENT}

This work is funded by the SRV-10/0026 Programme: Capacity Building and Technology Transfer for Mitigation of Geo-hazards in Vietnam in the Context of Climate Change.

\section{REFERENCES}

[1] Nidal Abid Al-Hamid Al-Dmour. TarffSim: Multiagent traffic simulation. European Journal of Scientific Research, 53(4):570-575, 2011.

[2] Ramachandran Balakrishna, Yang Wen, Moshe Ben-Akiva, and Constantinos Antoniou. Simulation-based framework for transportation network management in emergencies. Transportation Research Record, (2041):80-88, 2008.

[3] Jaime Barceló and Jordi Casas. Dynamic Network Simulation with AIMSUN. In Ryuichi Kitamura and Maso Kuwahara, editors, Simulation Approaches in Transportation Analysis, volume 31 of Operations Research/Computer Science Interfaces Series, pages 57-98. Springer US, 2005.

[4] M. Behrisch, L. Bieker, J. Erdmann, and D. Krajzewicz. SUMO simulation of urban mobility: An overview. In SIMUL 2011, The Third International Conference on Advances in System Simulation, pages 63 68, Barcelona, Spain, October 2011

[5] A. Caris, G. K. Janssens, and C. Macharis. Modelling complex Intermodal Freight flows, pages 291-300. Springer, Berlin/Heidelberg, 2009.

[6] MATSim development team (ed.). MATSIM-T: Aims,approach and implementation. Technical report, IVT, ETH Zrich, Zrich, 2007.

[7] Edsger. W. Dijkstra. A note on two problems in connexion with graphs. Numerische Mathematik, 1:269-271, 1959.

[8] Alexis Drogoul, Edouard Amouroux, Philippe Caillou, Benoit Gaudou, Arnaud Grignard, Nicolas Marilleau, Patrick Taillandier, Maroussia Vavasseur, Duc An Vo, and Jean-Daniel Zucker. GAMA: A Spatially Explicit, Multi-level, Agent-Based Modeling and Simulation Platform. In Yves Demazeau, Toru Ishida, Juan M. Corchado, and Javier Bajo, editors, International Conference on Practical Applications of Agents and Multiagent Systems (PAAMS), Salamanca, Spain, 22/05/201324/05/2013, volume 7879 of Lecture Notes in Computer Science, pages 271-274, http://www.springerlink.com, 2013. Springer.

[9] Rainer Frick. Simulation of transportation networks. In Proceedings of the 2011 Summer Computer Simulation Conference, SCSC '11, pages 188-193, Vista, CA, 2011. Society for Modeling \& Simulation International.

[10] Balaji Parasumanna Gokulan and Dipti Srinivasan. Multi-agent system in urban traffic signal control. IEEE Comp. Int. Mag., 5(4):43-51, 2010.

[11] Johan Holmgren, Paul Davidsson, Jan A. Persson, and Linda Ramstedt. Tapas: A multi-agent-based model for simulation of transport chains. Simulation Modelling Practice and Theory, 23:1-18, 2012.

[12] Michal Jakob, Zbyněk Moler, Antonín Komenda, Zhengyu Yin, Albert Xin Jiang, Matthew P. Johnson, Michal Pěchouček, and Milind Tambe. Agentpolis: towards a platform for fully agent-based modeling of multi-modal transportation (demonstration). In Proceedings of the 11th International Conference on Autonomous Agents and Multiagent Systems - Volume 3, AAMAS '12, pages 1501-1502, Richland, SC, 2012. International Foundation for Autonomous Agents and Multiagent Systems.
[13] Daniel Krajzewicz. Traffic Simulation with SUMO Simulation of Urban Mobility Fundamentals of Traffic Simulation. volume 145 of International Series in Operations Research \& Management Science, chapter 7, pages 269-293. Springer New York, New York, NY, 2010.

[14] Ulf Lotzmann. TRASS: A Multi-Purpose Agent-Based Simulation Framework for Complex Traffic Simulation Applications, pages 79-107. 2009.

[15] L. J. Manning, J. W. Hall, C. G. Kilsby, S. Glendinning, and M. G. Anderson. Spatial analysis of the reliability of transport networks subject to rainfall-induced landslides. Hydrological Processes, 22(17):3349-3360, 2008.

[16] David Meignan, Olivier Simonin, and Abderrafiaa Koukam. Simulation and evaluation of urban bus-networks using a multiagent approach. Simulation Modelling Practice and Theory, 15(6):659-671, July 2007.

[17] Dawit Mengistu, Peter Tröger, Lars Lundberg, and Paul Davidsson Scalability in distributed multi-agent based simulations: The jade case. In Proceedings of the 2008 Second International Conference on Future Generation Communication and Networking Symposia - Volume 05, FGCNS '08, pages 93-99, Washington, DC, USA, 2008. IEEE Computer Society.

[18] Manh Hung Nguyen and Tuong-Vinh Ho. Modelling circulation behaviour of vietnamese: Applying for simulation of hanoi traffic network. In Phan Cong Vinh, Vangalur S. Alagar, Emil Vassev, and Ashish Khare, editors, ICCASA, volume 128 of Lecture Notes of the Institute for Computer Sciences, Social Informatics and Telecommunications Engineering, pages 24-34. Springer, 2014.

[19] Manh Hung Nguyen, Tuong-Vinh Ho, Manh Son Nguyen, Thi Hoai Phuong Phan, Thi Ha Phan, and Van Anh Trinh. An agent-based model for simulation of traffic network status. In Lam Thu Bui, YewSoon Ong, Nguyen Xuan Hoai, Hisao Ishibuchi, and Ponnuthurai Nagaratnam Suganthan, editors, SEAL, volume 7673 of Lecture Notes in Computer Science, pages 218-227. Springer, 2012.

[20] Manh Hung Nguyen, Tuong Vinh Ho, and Tan Hiep Nguyen. On the dynamic optimization of traffic lights. Asian Simulation and Modeling, Mahidol University, pages 35-43, 2013.

[21] Manh Hung Nguyen, Manh Son Nguyen, Thi Ha Phan, and Van Anh Trinh. Dynamic path optimization in traffic routing. Asian Simulation and Modeling, Mahidol University, pages 43-51, 2013.

[22] Kalyan S. Perumalla. A systems approach to scalable transportation network modeling. In Proceedings of the 38th conference on Winter simulation, WSC '06, pages 1500-1507. Winter Simulation Conference, 2006.

[23] M. Piórkowski, M. Raya, A. Lezama Lugo, P. Papadimitratos, M. Grossglauser, and J.-P. Hubaux. TraNS: realistic joint traffic and network simulator for VANETs. SIGMOBILE Mob. Comput. Commun. Rev., 12(1):31-33, January 2008.

[24] Ana Lusa Ramos, Jos Vasconcelos Ferreira, and Jaume Barcel. Modeling \& simulation for intelligent transportation systems. International Journal of Modeling and Optimization, 2(3):274-279.

[25] Björn Schünemann. V2X simulation runtime infrastructure VSimRTI: An assessment tool to design smart traffic management systems. Comput. Netw., 55(14):3189-3198, October 2011.

[26] Yan Xu and Gary Tan. An offline road network partitioning solution in distributed transportation simulation. In Proceedings of the 2012 IEEE/ACM 16th International Symposium on Distributed Simulation and Real Time Applications, DS-RT '12, pages 210-217, Washington, DC, USA, 2012. IEEE Computer Society.

[27] Gregory Zacharewicz, Jean-Christophe Deschamps, and Julien Francois. Distributed simulation platform to design advanced rfid based freight transportation systems. Comput. Ind., 62(6):597-612, August 2011.

[28] Bo Zhang, Wai Kin (Victor) Chan, and Satish V. Ukkusuri. Agent-based discrete-event hybrid space modeling approach for transportation evacuation simulation. In Proceedings of the Winter Simulation Conference, WSC '11, pages 199-209. Winter Simulation Conference, 2011.

[29] Jiashun Zhang and Rongjie Lv. Multiagent competition simulation of integrated transportation system. TELKOMNIKA Indonesian Journal of Electrical Engineering, 11(1):345-350, 2013. 